\title{
Channel Optical Waveguides with Spatial Longitudinal Modulation of Their Parameters Induced in Photorefractive Lithium Niobate Samples
}

\section{A D Bezpaly and V M Shandarov}

Tomsk State University of Control Systems and Radioelectronics, Lenin Prospect 40, Tomsk, 634050, Russia

\section{Abstract}

The influence of point-by-point exposure of lithium niobate sample surface, doped with photorefractive impurities, to the longitudinal modulation of refractive index inside the formed waveguide channel is experimentally studied using optical probing method. Formation of channel waveguides by laser beam in different directions with

Corresponding Author:

A D Bezpaly

shandarovvm@svch.rk.tusur.ru

Received: 10 February 2018

Accepted: 14 April 2018

Published: 7 May 2018

Publishing services provided by Knowledge

(c) A D Bezpaly and V M

Shandarov. This article is

distributed under the terms of

the Creative Commons

Attribution License, which permits unrestricted use and redistribution provided that the original author and source are credited.

Selection and Peer-review under the responsibility of the RFYS Conference Committee.

\section{G OPEN ACCESS} respect to the crystal optical axis has been demonstrated. The channel waveguides have been formed via the point-by-point exposure at light wavelengths of $532 \mathrm{~nm}$ and $450 \mathrm{~nm}$. Variation of distance between exposing light spot centres demonstrates the possibilities of optical inducing of longitudinally homogeneous and inhomogeneous channel waveguides.

\section{Introduction}

The elements of laser light localization and light fluxes controllers like phase diffraction gratings and waveguide optical switches based on photorefractive materials are essential part of integrated optical circuits, devices and equipment of photonics [1-3]. One of the ways to form similar photonic elements in photorefractive crystals is optical inducing, which allows to set and manage different topologies during the formation of such structures $[4,5]$. The photorefractive sensitivity of lithium niobate $\left(\mathrm{LiNbO}_{3}\right)$, lithium tantalate $\left(\mathrm{LiTaO}_{3}\right)$, strontium-barium niobate $\left(\mathrm{Sr}_{x} \mathrm{Ba}_{1-x} \mathrm{Nb}_{2} \mathrm{O}_{6}\right)$ and some other crystals can be changed by several orders of magnitude, varying the concentration of impurities introduced when samples are doped through the surface or during the growth of crystals $[6,7]$.

The concentration raise of impurities introduced into the crystal during its growing is limited by decreasing of bulk sample mechanical properties due to the increase 
of defect numbers and appearance of elastic stresses. Modern technologies of the sold-state diffusion, the ion exchange and the ion implantation allow significantly increase the impurity concentration within material surface layers, additionally making it possible to introduce various impurities (or their combinations) into different regions of the sample surface [6 - 8]. Thus, the approach of surface doping makes possible varying the physical properties of material surface layer within its different areas over a wide range.

The main aim of this work is experimental studies of different channel waveguides including those with longitudinal modulation of their parameters, optically induced in lithium niobate sample with Cu-doped surface layer.

\section{Experimental setup and conditions}

Channel optical waveguides are induced within surface layer of photorefractive sample at its exposure with focused laser beam using the shift of the whole sample with respect to the light spot as it is shown in Figure 1. Laser beam with wavelengths $\lambda=532$ $\mathrm{nm}$ (solid-state YAG: $\mathrm{Nd}^{3+}$ laser with frequency doubling) or $\lambda=450 \mathrm{~nm}$ (semiconductor laser) is focused onto the doped crystal surface with 10x microscopic objective. The cross-sectional area of exposed surface is varied in different experiments from $\sim 300$ to $2000 \mu \mathrm{m}^{2}$. The dimensions of the sample used are $30 \times 3 \times 15 \mathrm{~mm}^{3}$ along $\mathrm{X}, \mathrm{Y}$, and $\mathrm{Z}$ axes. The sample has been thermally doped with Cu ions from a film deposited onto the wafer surface perpendicular to the crystal Y surface by vacuum sputtering. The thermal diffusion has been performed in air for 10 hours at $900^{\circ} \mathrm{C}$ temperature. The doped layer thickness is estimated from the doping conditions and known diffusion coefficient of $\mathrm{Cu}$ in $\mathrm{LiNbO}_{3}[9,10]$. It makes up about $100 \mu \mathrm{m}$. It has been revealed in [11] that $\mathrm{Cu}$ presence does not increase the refractive indexes of $\mathrm{LiNbO}_{3}$ that exclude formation of optical waveguides. Cu-doping does not also increase the dark conductivity of $\mathrm{LiNbO}_{3}$. It makes possible the long-time storage of photorefractive elements in this material.

It is known that photovoltaic mechanism of charge transport results in the decreasing of both, extraordinary and ordinary refractive indices under light influence $[5,6]$. Due to that the light trapping at $\mathrm{LiNbO}_{3}$ illumination can be observed in between exposed crystal areas with dropped refractive indices. The unexposed region with «increased» refractive index is bounded in the direction along crystal depth by the illuminated areas of the sample by the thickness of doped layer with photorefractive properties. 


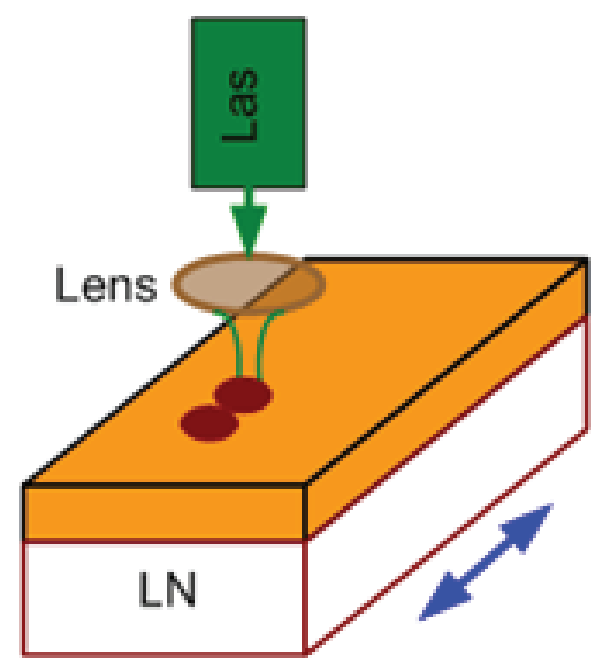

Figure 1: Experimental setup for the doped crystal surface exposure by focused laser beam.

The whole area of optically induced refractive index inhomogeneities is probed with collimated beam of the He-Ne laser like shown in Figure 2. Two exposed parallel stripes form channel waveguide between them. These stripes contain some exposed points with a distance between their centers from 20 to $50 \mu \mathrm{m}$ in dependence on light spot diameter on the exposed surface. The local refractive index change is studied by the scanning of focused laser beam of He-Ne laser, which probes optically uniform part (Figure 3 a), exposed stripe part (Figure 3 b), and the surface part between exposed stripes (Figure $3 \mathrm{C}$ ).

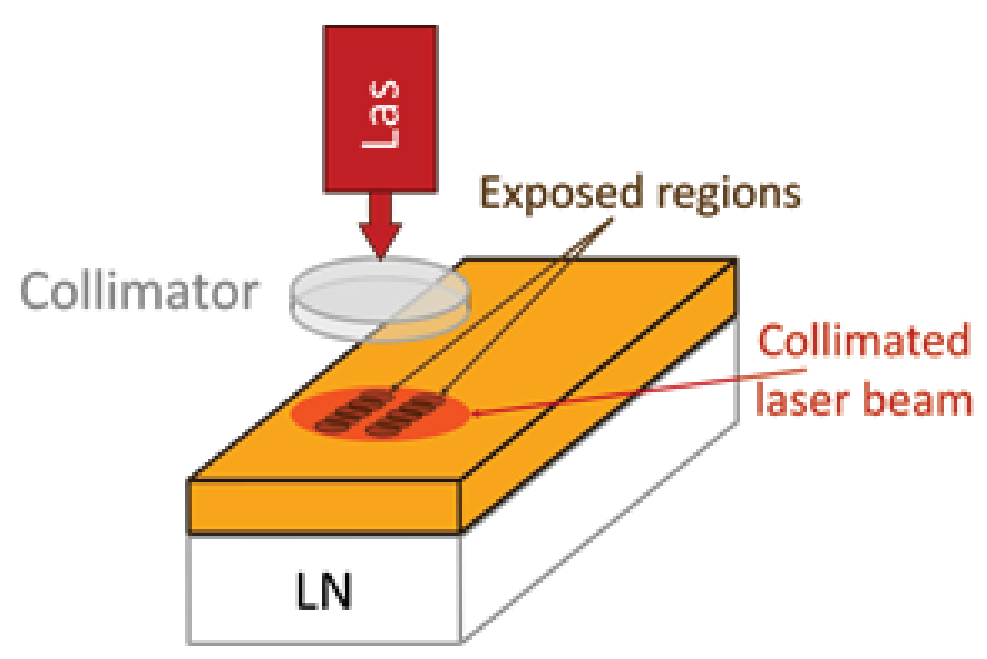

Figure 2: Schematic image of optically induced inhomogeneities study by optical probing.

The longitudinal modulation of refractive index change is also studied with the light diffraction method. Both, near field and far field diffraction images are studied. It should be noted, that far field diffraction images are formed by light beam focused 


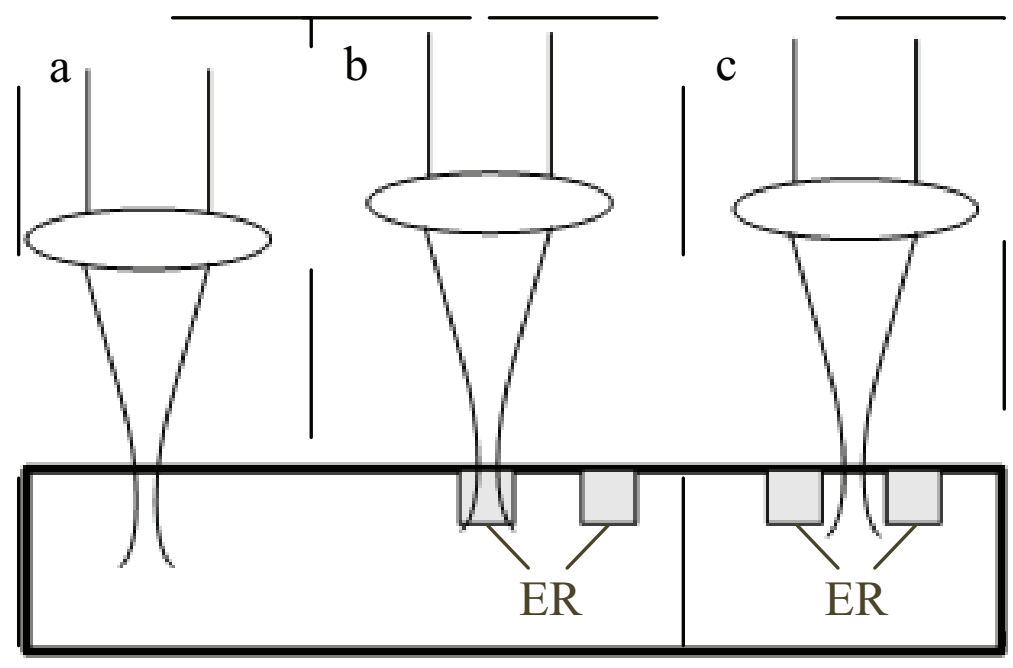

Figure 3: Sketch of experimental configurations at the diffraction study of light induced waveguide channels.

with cylindrical lens to decrease the optical power loss and to increase the diffraction efficiency.

\section{Experimental results and discussions}

Some particular results of optical probing of induced waveguide structures along direction normal to the sample surface are shown in Figure 4 and Figure 5. Light patterns in the cross-sections of laser beam probing the crystal surface in their different parts are studied using the laser beam analyzer.

Images in Figures 4 a, b, c illustrate light pictures in some intermediate zone of diffraction at optical probing of the sample surface by focused laser beam $(\lambda=633 \mathrm{~nm})$ with diameter $50 \mu \mathrm{m}$ in optically uniform part (Figure $4 \mathrm{a}$ ), in the areas of exposed stripe (Figure $4 \mathrm{~b}$ ), and between the exposed stripes (Figure $4 \mathrm{c}$ ). The exposure is performed using laser beam with wavelength of $532 \mathrm{~nm}$, its diameter at half maximum intensity $40 \mu \mathrm{m}$, light polarization corresponding to ordinary wave of crystal, and exposure time of $10 \mathrm{~s}$ for every point of the structure. The distance between adjacent light spots is $25 \mu \mathrm{m}$ onto the exposed surface.

The light pattern corresponding to the optical probing of optically uniform surface part demonstrates the circular symmetry. However, this symmetry cannot save when the focused beam probes exposed areas of the surface. When the laser beam probes the exposed stripe, this surface area acts as the defocusing cylindrical lens in the central part of light beam cross-section. It results in the redistribution of light intensity over the beam cross-section in direction parallel to the crystal optical axis. In turn, 
the area between exposed stripes act as the focusing cylindrical lens and changes the probe bam intensity distribution along the same optical axis direction, but in opposite way.

Optical probing of the whole exposed area is performed with $0.9 \mathrm{~mm}$ He-Ne laser beam diameter ( $\lambda=633 \mathrm{~nm}$ ). The near field diffraction images at the output surface of $\mathrm{LiNbO}_{3}$ :Cu sample are shown in Figure 5 . Polarization of the probing beam corresponds to the extraordinary wave of $\mathrm{LiNbO}_{3}$ that makes possible using its maximal electrooptic coefficient $\mathrm{r}_{33}[6,12]$. The channel waveguides have lengths of $0.5 \mathrm{~mm}$ and different longitudinal homogeneity. These waveguides have been formed by couples of parallel stripes with decreased refractive indices oriented normally to optical axis of the crystal. These are dark stripes with distances between exposed point centers 60 $\mu \mathrm{m}$ (Figure $5 \mathrm{a}$ ) and $25 \mu \mathrm{m}$ (Figure $5 \mathrm{~b}$ ) in the central part of these images. The bright areas between exposed stripes may operate as the channel optical waveguides when light propagates along $X$ direction of the crystal. The waveguide widths make up 20 $\mu \mathrm{m}$ in the cases illustrated with these images.
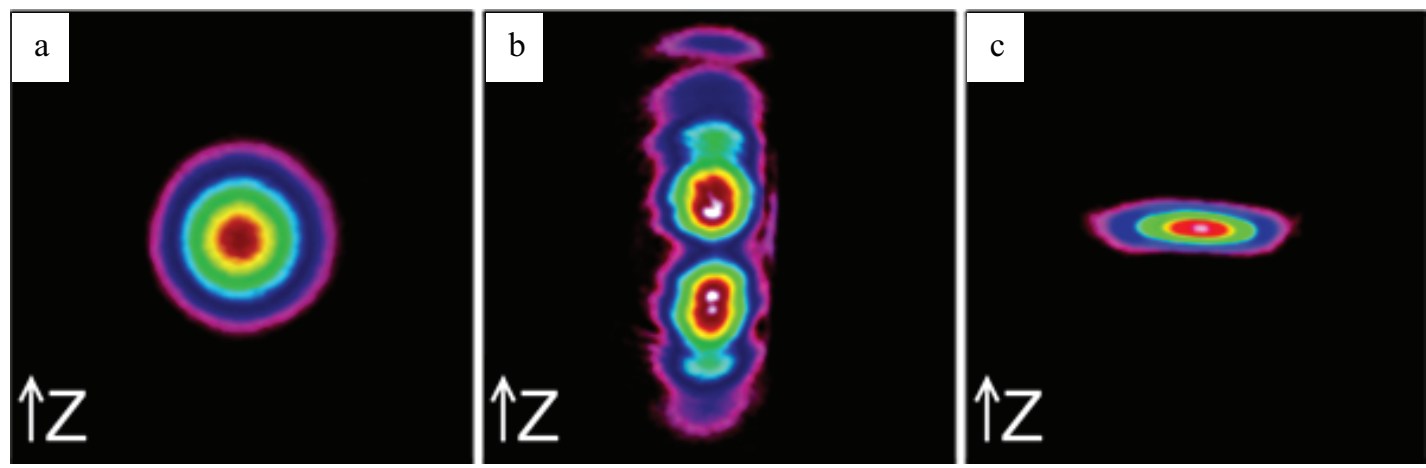

Figure 4: Light images at optical probing of optically uniform part of the sample (a), the exposed stripe (b) and the area between exposed stripes (c).

As it has been noted we form the waveguide channel structures oriented parallel to the crystal optical axis in lithium niobate using the point-by-point photorefractive exposure processing at the first time to our knowledge. Formation of similar structures with uniform light stripes oriented along direction of charge carrier transport is forbidden for lithium niobate [6]. It is because the electric space charges appear in this case only at the boundaries of illuminated region normal to the optical axis direction of the crystal. It results in the low space charge electric field and very small refractive index changes induced in lithium niobate at such illumination. However, the sufficient refractive index changes can arise if the illumination is spatially modulated. It is illustrated by the near field diffraction patterns in Figure 6 obtained at the scanning of crystal sample surface along the optical axis direction with light spots with different 


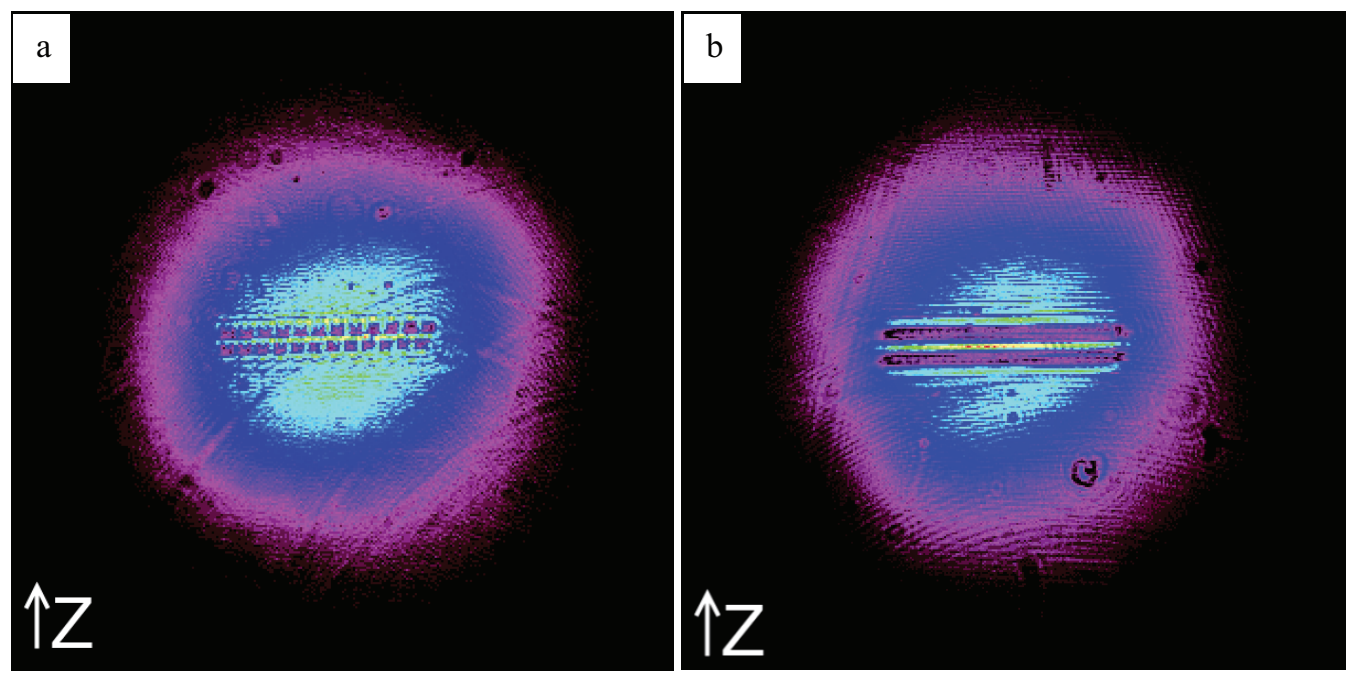

Figure 5: Near field light patterns at optical probing of the induced channel waveguide area with different longitudinal homogeneity (different distances between centers of exposing light spots).

separation of light spots. Besides formation of channel optical waveguides, we can also modulate their parameters along this direction with necessary rules.

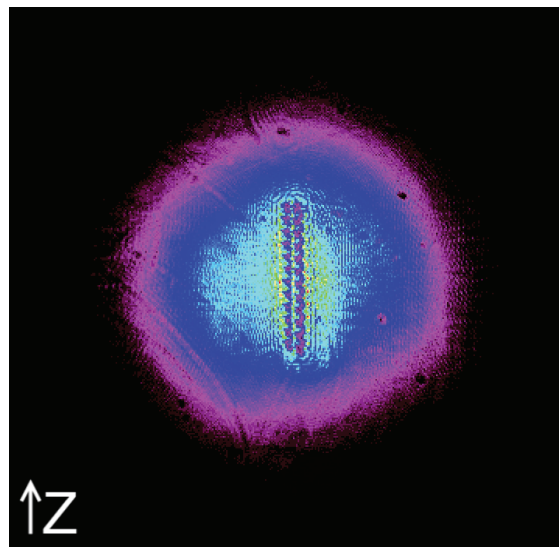

a

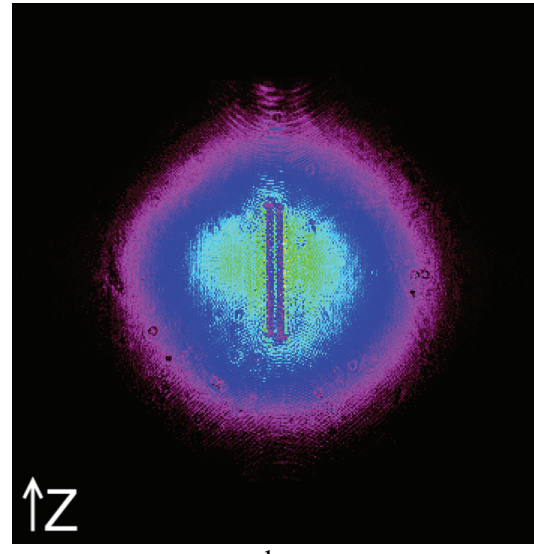

$\mathrm{b}$

Figure 6: Near field light patterns at optical probing of the induced channel waveguide area at their orientations along crystal optical axis and spatial separation of exposing spot centers $60 \mu \mathrm{m}(a)$ and $20 \mu \mathrm{m}$ (b).

The spatial periodic modulation of channel waveguide parameters results in the different shapes of diffraction images in far field. It is illustrated by images in Figure 7 which correspond to the diffraction of elliptically shaped light beam $(\lambda=633 n \mathrm{n})$ on channel waveguide structures with non-uniform (a) and almost uniform (b) refractive index change along the waveguide direction normal to the crystal optical axis.

Picture in Figure 7 a shows intensity profile of diffraction pattern in far field along direction of exposed stripe. It illustrates the periodic refractive index change inside the material in direction along the exposed strip (the distance between the centers of 


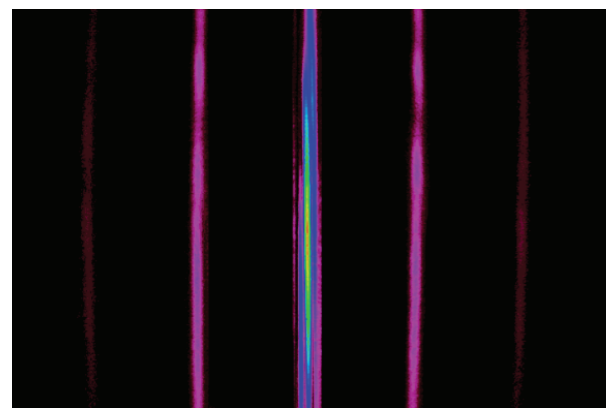

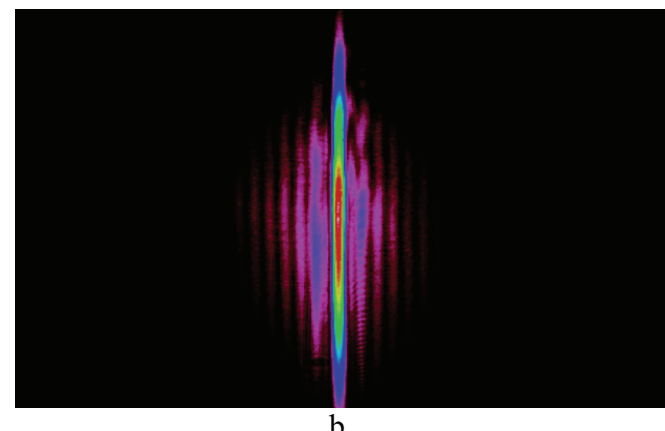

b

Figure 7: Far field light patterns at optical probing of the induced channel waveguide area at their normal orientation along crystal optical axis and spatial separation of exposing spot centers $60 \mu \mathrm{m}$ (a) and $25 \mu \mathrm{m}$ (b).

light spots at the crystal surface is $60 \mu \mathrm{m}$ ). This periodicity leads to the appearance of diffraction maxima, the angular deviation of which from direction of transmitted without diffraction beam is determined by distance between the centers of exposed points. The light intensity distribution is symmetrical in this case along waveguide direction and at least two orders of diffraction maxima are present in the light pattern that points to the limited thickness of waveguide structure.

Figure $7 \mathrm{~b}$ corresponds to the diffraction of light in almost longitudinally homogeneous exposed regions with a distance between the centers of exposed points of 25 $\mu \mathrm{m}$. In this case the refractive index change along the exposed stripe is practically uniform due to the small distance between exposing spot centers and saturable optical nonlinearity in photorefractive lithium niobate. Because of that there is in the far field only transmitted without diffraction beam with low noise at its sides due to the light diffraction on waveguide structure boundaries normal to the crystal optical axis. The intensity distribution profile corresponds here to almost longitudinally homogeneous refractive index change of formed waveguide structure.

To check finally the waveguide properties of optically induced structures, we excite light in waveguides along their directions. Light beam of He-Ne laser $(\lambda=633 \mathrm{~nm})$ is focused onto the entrance surface of waveguide channel using spherical lens with focal length of 3 centimeters. The light picture at the exit surface of the waveguide is illustrated by image in Figure 8 a. Large transverse size of waveguide channel ( $\sim 20$ $\mu \mathrm{m}$ ) results in propagation within this the modes of higher numbers, not only the mode of zero order. It is clearly seen from light pattern (Figure 8 a) and its intensity profile (Figure 8 b). 

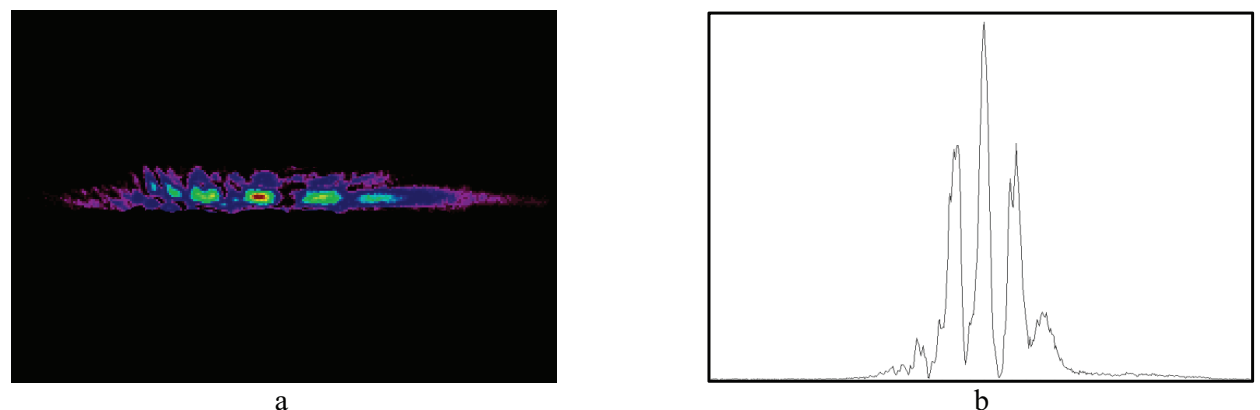

Figure 8: The light pattern at the output facet of channel waveguide (a) and intensity profile (b) of this pattern along the crystal optical axis (b).

\section{Conclusion}

In conclusion, the possible formation of optically reconfigurable channel waveguides within lithium niobate samples with surface layers doped with photorefractive impurities is experimentally demonstrated in this work. Waveguide configuration is not limited by simple straight elements, the waveguide channels may be longitudinally modulated with their parameters or curvature. Besides, point-by-point exposure allows formation of waveguide channel structures oriented along forbidden directions. Such optically controlled and reconfigured elements are perspective components of modern photonic devices.

\section{Acknowledgments}

This study was carried out with the financial support of Ministry of Education and Science of Russia (the project on request 3.1110.2017/PCh).

\section{References}

[1] Hirohito Yamada, Tao Chu, Satomi Ishida and Yasuhiko Arakawa 2006 IEEE Journal of Selected Topics in Quantum Electronics vol 12 pp 1371-1379

[2] Dominik G Rabus, Zhixi Bian, and Ali Shakouri 2007 IEEE Journal of Selected Topics in Quantum Electronics vol 13 Pp 1249-1256

[3] Marco Bazzan and Cinzia Sada 2015 Applied Physic Reviews vol 2 pp 040603-1 040603-25

[4] Trenikhin P, Shandarov V and Chen F 2011 Doklady TUSURa № 2 (24) Part 2 pp 131-134

[5] Kanshu A et al. Appl. Phys. B. 2009 vol 95 № 3 Pp 537-543

[6] Kip D Appl. Phys. B. 1998 vol 67 P 131 
[7] Chen F J. Appl. Phys. 2009 vol 106 N 8 p 081101

[8] Das B K Optics Letters 2004 vol 29 p 165

[9] Peithmann K Hukriede J Buse K and Kraetzig E Phys. Rev. B. 2000 vol 61 pp 46154620

[10] Imbrock J Wirp A Kip D Kraetzig E and Dirk B J. Opt. Soc. Am. B. 2002 vol 19 pp 18221829

[11] Popov V and Shandarov V Sov. Phys. Tech. Phys. 1991 vol 36 pp 1380-1382

[12] Krätzig E Ferroelectrics 1990 vol 104 Pp 257-268 